\title{
Synthèse
}

\section{Physiologie des interactions entre pomme de terre et pucerons : vers une nouvelle stratégie de lutte basée sur les systèmes de défense de la plante}

\author{
Nicolas Harmel ${ }^{1}$ \\ Frédéric Francis ${ }^{1}$ \\ Eric Haubruge ${ }^{1}$ \\ Philippe Giordanengo ${ }^{2}$ \\ ${ }^{1}$ Faculté universitaire des sciences \\ agronomiques de Gembloux, \\ 2, passage des Déportés, \\ 5030 Gembloux \\ Belgique \\ <harmel.n@fsagx.ac.be> \\ $<$ francis.f@fsagx.ac.be> \\ <haubruge.e@fsagx.ac.be> \\ 2 Université de Picardie Jules Verne, \\ EA3900-BioPl, \\ Biologie des plantes et contrôle des insectes \\ ravageurs (BioPI), \\ 33, rue Saint-Leu, \\ 80039 Amiens cedex 01 \\ France \\ <philippe.giordanengo@u-picardie.fr>
}

\begin{abstract}
Résumé
La culture de pommes de terre est soumise à de nombreux ravageurs parmi lesquels les pucerons ne sont pas les moindres. Ils exercent une action directe par prélèvement de sève sur le végétal mais surtout indirecte en véhiculant de nombreux virus phytopathogènes. L'utilisation massive de produits aphicides a conduit à la sélection de populations de pucerons résistants. Comme la découverte et le développement de nouveaux insecticides chimiques sont désormais en nette diminution, il reste peu d'alternatives pour lutter contre les pucerons qui sont maintenant résistants à plusieurs familles d'insecticides. Consciente de cette problématique, la communauté scientifique a adopté une stratégie de développement de moyens de lutte biologique tenant compte des défenses naturelles des plantes contre les pucerons. Les différentes stratégies de lutte envisageables sont présentées et développées dans cet article.
\end{abstract}

Mots clés : lutte biologique ; mécanisme de défense ; puceron ; Solanum tuberosum.

Thèmes : métabolisme ; pathologie ; productions végétales.

\begin{abstract}
Fighting potato aphids: Alternatives to insecticides based on plant insect interaction physiology

Potato crops are facing too many pests, among which aphids are one of the most important. They are responsible for direct (phloem intake) and indirect damage the consequences of which are destructive: the injected saliva can be the vector of many pathogenic viruses. Intense use of aphicides has led to the selection of resistant aphid populations. As new chemical discovery and development are decreasing, few alternatives are available. Conscious of this problem, the scientific community is following a strategy consisting in developing biological control taking into account natural plant defences against aphids which have become multiresistant to insecticides. Conceivable strategies are presented and developed in this paper.
\end{abstract}

Keywords: biological control; defence mechanisms; aphids; Solanum tuberosum.

Subjects: metabolism; pathology; vegetal productions. a pomme de terre Solanum tuberosum L., cultivée pour son tubercule, est une culture d'importance mondiale pour l'alimentation humaine et l'industrie. Il en existe plus de 3000 variétés présentant des propriétés diverses en termes de qualité commerciale, de résistance aux maladies, de productivité ou encore d'aptitude à la transformation et à la conservation. Cette culture est soumise à la pression d'une multitude de bioagresseurs (champignons, bactéries, virus, nématodes et insectes) pouvant soit occasionner des pertes sévères en rendement, soit altérer la qualité des productions de tubercules ou de plants (semence) qui se traduisent par un déclassement, voire une non-commercialisation. Parmi les insectes 
ravageurs, les pucerons occupent une très large place. Appartenant à l'ordre des Hémiptères Sternorrhynques, les pucerons sont répartis en plus de 4000 espèces. Ces ravageurs fortement dommageables pour une multitude d'espèces végétales résistent à de nombreuses familles d'insecticides. Il est donc primordial d'adopter des alternatives novatrices et efficaces aux insecticides en culture de pomme de terre. Le puceron de la pomme de terre Macrosiphum euphorbiae, le puceron vert du pêcher Myzus persicae, le puceron du cotonnier Aphis frangulae et le puceron du nerprun Aphis nasturtii posent réellement un problème en culture de pomme de terre. Une des stratégies envisageables repose sur l'exploitation des résistances naturelles de la plante et des faiblesses de l'insecte. Le présent article se focalise sur les relations physiologiques entre pucerons et pommes de terre, sur les composés végétaux dont l'efficacité a été testée contre les pucerons et sur les voies de biosynthèse de ces derniers pouvant être ciblées.

\section{Dégâts occasionnés par les pucerons}

Insectes de type piqueurs-suceurs, les pucerons s'alimentent exclusivement aux dépens de la sève élaborée et occasionnent des dommages tant directs qu'indirects aux plantes.

\section{Dégâts directs}

Les stylets du puceron lui permettent d'effectuer des piqûres dans la plante pour atteindre les tubes criblés. La perforation des feuilles et l'injection d'une salive liquide dans les tissus végétaux peuvent avoir des effets toxiques responsables de perturbations de nature physiologique se traduisant par des crispations, des dépigmentations des feuilles, la formation de galles. La croissance d'une plante fortement infestée peut également être perturbée suite au prélèvement de nutriments par les pucerons (Miles, 1989).

\section{Dégâts indirects}

Le miellat excrété par les pucerons, bien que non toxique, constitue un milieu favorable au développement de microorganismes, notamment des champi- gnons saprophytes responsables des fumagines, et peut également induire des phénomènes de dessiccation (Huang et al., 1983).

Les aphides véhiculent de nombreux virus et sont susceptibles de causer jusqu'à $20 \%$ de pertes en rendement dans le Nord de la France (Duvauchelle et Dubois, 1997). $M$. persicae peut transmettre plus d'une centaine de virus alors que M. euphorbiae peut en véhiculer une quarantaine. Les virus les plus connus sur pomme de terre et transmis par les pucerons sont le virus de l'enroulement, les virus Y, A, S, X et M (Kennedy et al., 1963). Les dégâts causés sont localisés au niveau des feuilles, avec l'apparition de taches nécrotiques, de mosaïques, d'enroulement ou de dépigmentations foliaires. Certains virus induisent également des nécroses au niveau des tubercules qui les rendent non commercialisables.

\section{Moyens de lutte}

Outre les moyens classiques (suivi et élimination de toute source de contamination du vecteur, défanage plus ou moins précoce, limitation de l'enherbement...), l'utilisation d'insecticides de synthèse est largement répandue. Autrefois, ces ravageurs étaient combattus en augmentant les doses des pesticides utilisés ou en appliquant de nouvelles matières actives. Ces deux stratégies sont aujourd'hui révolues. L'utilisation massive de produits aphicides a conduit à la sélection de populations de pucerons résistants. Ainsi, $M$. persicae est résistant à plusieurs familles d'insecticides par surproduction d'estérases (contre les carbamates et les organophosphorés) ou mutation des canaux à sodium (contre le DTT et les pyréthrinoïdes) (Devonshire et al., 1998 ; Foster et al., 2003). De plus, l'utilisation de quantités croissantes d'insecticides chimiques représente un risque pour la santé humaine et pour l'environnement. Les contraintes législatives se durcissent par de nouvelles réglementations relatives à l'homologation et à l'utilisation des substances chimiques telles que le retrait prochain du marché de plusieurs molécules actives. Ces nouvelles contraintes en phytoprotection constituent de réels enjeux technologiques, économiques et écologiques. Le développement de nouvelles stratégies de phytoprotection intègre nécessairement la prise en compte des risques vis-à-vis de l'homme et de l'environnement.

\section{Quelles sont donc les alternatives?}

Il convient dès lors de développer des méthodes de lutte alternatives, novatrices et efficaces. Parmi celles-ci, la lutte biologique avec l'utilisation d'insectes aphidiphages tels que des prédateurs ou des insectes hyménoptères parasitoïdes a été envisagée. Le recours à certains pathogènes (virus, bactéries ou champignons entomopathogènes) est également un moyen de lutte contre les pucerons (Ying et al., 2003). Une autre stratégie repose sur l'exploitation des résistances naturelles des plantes. L'abondance et la diversité des métabolites secondaires produits par les plantes représentent une source importante de molécules qui doivent dès aujourd'hui faire l'objet d'un investissement majeur dans le domaine de la recherche.

La colonisation d'une plante par un puceron s'effectue en deux étapes. Il doit d'abord localiser la plante puis, lorsque l'insecte entre en contact avec celle-ci, l'accepter comme hôte. Une fois posé sur une plante, un aphide arrête ses mouvements ou les restreint à une petite zone. Plusieurs types d'informations lui parviennent alors et, à chaque étape du processus comportemental, il prend la décision de poursuivre ses investigations ou de quitter la plante, jusqu'à l'acceptation finale. Celle-ci a lieu lorsqu'il effectue une prise alimentaire prolongée ou une ponte. La première étape de la séquence comportementale débute par la perception des composés volatils émis par les plantes, puis des propriétés physicochimiques de surface. Ensuite, les pucerons testent les composés internes de la plante par des piqûres de sondages jusqu'à atteindre le phloème où circule la sève élaborée dont ils se nourrissent (Schoonhoven et al., 1998). Cette chronologie du processus de sélection et d'acceptation de la plante hôte nous servira de fil conducteur. Enfin, après avoir évoqué les facteurs de résistance végétaux efficaces contre les pucerons, nous présenterons certaines de leurs fonctions physiologiques clés pouvant être ciblées.

\section{Substances volatiles émises par la pomme de terre}

Les pucerons peuvent détecter les odeurs émises pas les plantes grâce à leurs anten- 
nes (Storer et al., 1996). De plus, les substances volatiles émises en surface des plantes peuvent influencer les processus d'acceptation par les aphides (Pickett et al., 1992). D'après Paré et Tumlinson (1999), les quantités de composés volatils diffusés par les plantes sont augmentées suite à l'attaque d'insectes phytophages, et la composition de ce bouquet odorant est spécifique du couple plante-insecte phytophage. Certains composés peuvent être attractifs, comme par exemple les volatils de S. tuberosum infestee par le virus de l'enroulement vis-à-vis de $M$. persicae, les pucerons étant préférentiellement attirés par des plants atteints par ce virus en comparaison à des plants sains ou atteints par d'autres viroses (Eigenbrode et al., 2002). Par contre, d'autres composés jouent le rôle de substances répulsives contre les aphides comme le $E$ - $\beta$-farnésène (EBF) produit en grande quantité par les trichomes glandulaires de Solanum berthaultii (Hawkes) (Gibson et Pickett, 1983). L'hexanal et le 3-hexénal, deux composés chimiques du bouquet "odeur verte" des feuilles, ont un impact négatif sur la fécondité de Myzus nicotianae (Hildebrand et al., 1993). Les substances volatiles interviennent également en tant que défenses indirectes pour la plante en attirant des insectes prédateurs ou parasitoïdes des pucerons. Cela a été vérifié pour la coccinelle prédatrice Coccinella septempunctata (Ninkovic et al., 2001), les larves et les adultes prédateurs d'Adalia bipunctata (Francis et al., 2004), Episyrphus balteatus, une autre espèce aphidiphage (Francis et al., 2005) et le parasitoïde Aphidius ervi (Du et al., 1998). Les molécules volatiles émises peuvent aussi agir comme signaux d'avertissement entre les plantes (Paré et Tumlinson, 1999).

\section{Facteurs}

\section{physicochimiques} de surface impliqués dans l'acceptation de la plante hôte

Des facteurs physiques comme une importante pubescence peuvent réduire l'acceptation de la plante hôte. La stratégie de résistance connue vis-à-vis des aphides est constituée par les trichomes glandulaires présents chez certaines Solanum sauvages. Ce système, contrôlé par plusieurs gènes et très étudié chez $S$. berthaultii (Tingey et Laubengayer, 1981), confère à ces plantes une antixénose en surface et allie à la fois résistance physique et chimique. Les exsudations d'ester de saccharose produites par les trichomes de type B peuvent se coller sur les tarses des aphides et perturber les processus comportementaux d'acceptation de la plante (Neal et al., 1990). Des sesquiterpènes volatils composés d'EBF sont également relargués par ces trichomes et ont pour effet de perturber le comportement de l'insecte qui tente de fuir. Le puceron rompt alors la tête d'un trichome de type A, ce qui a pour effet le relarguage de composés phénoliques (Avé et al., 1987). Ces produits entrent en contact avec des polyphénols oxydases et des péroxydases qui réagissent en un processus d'oxydation pour donner des quinones, formant un composé brun poisseux, collant, qui s'accumule sur le corps de l'insecte (Vallejo et al., 1994). Celui-ci est piégé, immobilisé, cesse de s'alimenter et meurt.

\section{Composés internes de la plante}

Une fois toutes les propriétés physicochimiques de surface perçues et analysées, les aphides insèrent leurs stylets dans les tissus de la plante qui progressent entre les cellules jusqu'à atteindre le phloème, site de prise alimentaire. Au cours de ce transit, les aphides effectuent des ponctions intercellulaires, mais également intracellulaires dans la plupart des cellules rencontrées. Ces prélèvements, qui s'accompagnent d'une injection de salive sont déterminants dans le choix de la plante hôte (Powell et al., 2006). Selon Tjallingii et Hogen Esch (1993), les cellules proches des vaisseaux phloémiens sont beaucoup plus ponctionnées que celles de l'épiderme ou du mésophylle, ce qui indiquerait que la recherche du phloème s'effectue par échantillonnage et que le puceron est capable de reconnaître la composition chimique des différents types de cellules rencontrées. Les aphides sont sensibles à certains types de composés qui jouent le rôle soit de stimulants, soit de composés inhibiteurs ou anti-appétants.

Parmi les composés internes présentés ci-après, seuls des inhibiteurs de protéases
(IP) et des lectines ont été identifiés dans la sève phloémienne de la plante qui est l'aliment des pucerons (Kehr, 2006).

\section{Métabolites secondaires}

Les métabolites secondaires sont des molécules qui, chez la plante, ont une fonction défensive induite ou constitutive et n'interviennent pas directement dans les processus de croissance et de développement et représentent de 1 à $3 \%$ de la matière sèche (Harbone, 1993).

Les glycoalcaloïdes (GA) constituent un groupe important de métabolites secondaires produits par les Solanaceae (Vaananen et al., 2000). Chez la pomme de terre, les GA sont synthétisés principalement dans les feuilles où ils s'accumulent mais ils sont également présents, en moindre proportion, dans les tubercules (Lachman et al., 2001). Ils sont considérés comme toxiques pour la santé humaine à des seuils de $20 \mathrm{mg} / 100 \mathrm{~g}$ de poids frais de tubercule (Friedman et McDonald, 1997). C'est pourquoi, la faible teneur en alcaloïdes a été privilégiée lors de programmes de sélection dans le cadre de l'amélioration génétique des Solanaceae afin d'atteindre des taux de 2 à $10 \mathrm{mg} / 100 \mathrm{~g}$ de poids frais (Esposito et al., 2002).

La solanine et la chaconine représentent plus de $95 \%$ des GA chez S. tuberosum (Lachman et al., 2001). Les GA exercent des effets répulsifs (antixénose) ou toxiques (antibiose) sur certains ravageurs et présentent donc un intérêt évident lorsqu'ils ne sont synthétisés que dans les feuilles (Rangarajan et al., 2000).

In planta, des teneurs élevées en GA foliaires semblent inefficaces vis-à-vis des pucerons $M$. persicae et Aulacorthum solani (Flanders et al., 1992) alors qu'in vitro certains GA présentent des effets toxiques pour $M$. euphorbiae (Güntner et al., 2000) et M. persicae (Fragoyiannis et al., 1998).

\section{Inhibiteurs de protéases}

Les plantes contiennent parfois de grandes quantités d'IP dans la sève phloémienne (Kehr, 2006). Ceux-ci peuvent se lier aux protéases digestives des insectes pour former des complexes stables et ainsi inhiber leur activité. Les hémiptères utilisent essentiellement des protéases à cystéines (Wolfson et Murdock, 1990). Bien qu'il existe des activités protéasiques dans les structures digestives des pucerons, celles-ci sont faibles (Deraison et al., 2004) et, à ce jour, aucune preuve 
n'a pu être apportée quant à l'existence de protéases digestives chez ces insectes. Les protéines ingérées seraient très peu hydrolysées et les protéines seraient capables de traverser l'épithélium digestif (Azzouz et al., 2005). Ainsi, Rahbé et al. (2003b) décrivent le passage d'IP dans l'hémolymphe des pucerons.

Strictement phloémophage, les pucerons prélèvent une partie des acides aminés dans le flux de sève, les bactéries endosymbiontes assurant par néosynthèse le complément en acides aminés essentiels. Ainsi, il paraît peu probable que les IP aient un effet sur ces insectes (Haq et al., 2004). Cependant, quelles que soient les cibles et les modalités d'action, différents travaux rapportent des effets aphicides de l'oryzacystatine I, un IP à cystéines (Rahbé et al., 2003a) et d'un inhibiteur de sérine protéase à affinité trypsique et chymotrypsique du pois (Rahbé et al., 2003b).

\section{Lectines}

Les lectines sont une classe de protéines possédant au moins un domaine non catalytique qui se lie de manière spécifique et réversible aux mono- ou oligosaccharides (Carlini et Grossi-de-Sa, 2002). Cette liaison provoque l'agglutination des cellules ou la précipitation des glycoconjugués. Chez les plantes supérieures, elles jouent essentiellement un rôle de défense naturelle et sont concentrées plus particulièrement dans les tissus de réserve, mais aussi dans les racines, les feuilles et la tige (Van Damme et al., 1997). Le mode d'action précis des lectines dans l'insecte demeure inconnu (Carlini et Grossi-de-Sa, 2002).

La concanavaline A, une lectine spécifique de glucose/mannose de Canavalia ensiformis, a un impact négatif sur les performances de $M$. persicae (Gatehouse et al., 1999) et d'Acyrthosiphon pisum (Sauvion et al., 2004) lorsqu'elle est administrée dans des diètes artificielles ou exprimée in planta chez des lignées de pommes de terre transgéniques.

Il y a quelques années, le débat des OGM et de l'utilisation des lectines est arrivé sur le devant de la scène publique à cause des effets négatifs supposés sur les ennemis naturels des insectes se nourrissant de ravageurs intoxiqués par des lectines et de certaines recherches démontrant les effets négatifs d'OGM exprimant une lectine sur la croissance de rats de laboratoire (Ewen et Pusztai, 1999). Couty et al. (2002) ont depuis montré que la lectine de perce-neige, Galanthus nivalis agglutinine, n'a aucun effet sur la mortalité et sur les performances d'apprentissage des hyménoptères, bien qu'un effet indirect sur le développement des parasitoïdes de pucerons A. ervi et Aphelinus abdominalis soit dû à la réduction de taille et/ou à la mortalité précoce des pucerons. Cette lectine n'a pas d'effet délétère sur $A$. bipunctata (Down et al., 2003).

\section{Facteurs de résistance}

Aucun gène de résistance n'a été décrit dans le genre Solanum; seul le gène $\mathrm{Mi}-1$ provenant d'une espèce sauvage de tomate et induisant une résistance au puceron $M$. euphorbiae, à deux espèces d'aleurodes et à trois espèces de nématodes Meloidogyne spp., a été cloné au sein de la famille des Solanacées (Kaloshian et Walling, 2005 ; Martinez de Ilarduya et al., 2003). Ce gène de type NBS/LRR est spécifique de certains clones de M. euphorbiae vis-à-vis desquels il induit une antixénose phloémienne (Goggin et al., 2001). La résistance semble être de type gène pour gène comme pour la résistance aux pathogènes (Kaloshian et Walling, 2005).

\section{Salive et éliciteurs}

Enfin, la salive des insectes joue un rôle primordial dans les relations entre la plante et l'insecte. Des éliciteurs de réactions de défense ont été identifiés dans la salive de chenilles de plusieurs espèces de Lépidoptères. On retrouve la $\beta$-glucosidase dans la salive de Pierisbrassicae L. (Mattiaci et al., 1995), le glucose oxydase dans la salive de Helicoverpa zea (Eichenseer et al., 1999) et la volicitine dans la salive de Spodoptera exigua Hübner (Alborn et al., 1997). À ce jour, aucun éliciteur n'a été identifié dans la salive des pucerons. Cependant, il a été montré que des plants de pomme de terre infestés par des pucerons se défendaient différemment que des plantes blessées mécaniquement par de simples aiguilles (Harmel et al., 2007). Il paraît donc tout à fait intéressant d'investiguer le protéome de la salive du puceron $M$. persicae à la recherche d'un éliciteur des réactions de défense (Francis et al., 2006 ; Harmel et al., 2008). Des enzymes intervenant certainement dans la détoxication des défenses de la plante ont été identifiés chez Schizaphis graminum, A. pisum et $M$. persicae par Cherqui et Tjallingii (2000), leur rôle devant encore être précisé. L'identification chez le puceron d'un composé salivaire induisant les défenses naturelles de la plante ouvrirait une nouvelle piste de développement de moyens de lutte alternatifs.

\section{Chitinases}

Chez les insectes, la chitine est un composé majeur de la cuticule et de la matrice péritrophique et joue le rôle d'un composé structural dynamique et protecteur. Chez ces invertébrés, les processus de croissance et de métamorphose dépendent de leur capacité à lyser puis resynthétiser les structures contenant de la chitine. L'importance de la chitine dans le développement de l'insecte conjuguée à l'absence de polymères de chitine chez les vertébrés a conduit les chercheurs à considérer son métabolisme comme cible potentielle de molécules insecticides. Ainsi, Fitches et al. (2004) ont montré que la chitinase synthétique de Lacanobia oleracea, la noctuelle de la tomate, était toxique pour cet insecte par administration orale. En revanche, une chitinase du coléoptère chrysomélide Phaedon cochleariae et une chitinase de la bactérie Serratia marcescens, respectivement délivrée in planta et in vitro, conduisent à une réduction de la mortalité larvaire et une augmentation du taux intrinsèque d'accroissement naturel des populations de M. persicae (Saguez et al., 2005). Cependant, la stratégie des chitinases n'en demeure pas moins une cible d'intérêt. En effet, le test d'inhibiteurs de chitinase de différentes natures et structures a montré de puissants effets aphicides de certains d'entre eux en provoquant de très fortes mortalités larvaires des pucerons intoxiqués (Saguez et al., 2006).

\section{Perturbation de I'hormone juvénile}

L'hormone juvénile a pour principales fonctions d'assurer le maintien des caractères juvéniles lors des mues larvaires et de stimuler la maturation du système reproducteur chez l'insecte adulte. Dès 
lors, la perturbation des fonctions de cette hormone permettrait d'entraîner certaines modifications létales lors du développement de l'insecte et, de cette manière, pourrait contribuer au contrôle des pucerons. Une des possibilités porte sur une enzyme clé de la biosynthèse de l'hormone juvénile: la farnésyl diphosphate synthase (Kikuchi et al., 2001). Une étude visant à inhiber la biosynthèse de cette hormone est actuellement en cours chez le puceron (Vandermoten et al., 2008).

\section{Conclusion}

Comme on peut s'en rendre compte, les alternatives basées sur la physiologie de l'interaction entre pucerons et pommes de terre sont nombreuses, certaines semblant plus réalistes et prometteuses que d'autres, leur étude n'étant pas au même niveau d'avancement. Les défenses végétales sont constituées par les substances volatiles, les facteurs physicochimiques de surface, les composés internes et les facteurs de résistance. Tous ces composés présentent une activité aphicide le plus souvent seulement en conditions artificielles (tests in vitro). Des stratégies ciblant la chitinase et l'hormone juvénile des pucerons et visant à étudier le contenu de leur salive sont actuellement en cours de développement. Il ne faut pas oublier que pour pouvoir être un jour commercialisé, un produit aphicide doit, en plus de son efficacité, répondre à de nombreux critères toxicologiques comme l'impact sur les organismes non ciblés puisque certains de ces organismes peuvent être utilisés comme auxiliaires de lutte. La lutte, basée sur l'utilisation de molécules issues des biotechnologies, ne constituera pas l'unique moyen de lutte en agriculture mais devra plutôt être considérée comme une stratégie de lutte à utiliser de manière raisonnée avec d'autres moyens déjà existants.

\section{Remerciements}

Nicolas Harmel est financé par une bourse des Fonds pour la formation à la Recherche dans l'industrie et dans l'agriculture (FRIA), Belgique. Les auteurs remercient le Fond national pour la recherche scientifique (FNRS), Belgique (FRFC numéros 2.4.561.06 et 2.4.586.04).

\section{Références}

Alborn T, Turlings TCJ, Jones TH, et al. An elicitor of plant volatiles from beet armyworm oral secretion. Science $1997 ; 276$ : 945-9.

Avé DA, Gergoy P, Tingey WM. Aphid repellent sesquiterpenes in glandular trichomes of
Solanum berthaultii and Solanum tuberosum. Entomol Exp Appl 1987 ; 44 : 131-8.

Azzouz H, Campan EDM, Cherqui A, et al. Potential effects of plant protease inhibitors, oryzacystatin I and soybean Bowman-Birk inhibitor, on the aphid parasitoid Aphidius ervi Haliday (Hymenoptera, Braconidae). J Insect Physiol $2005 ; 51: 941-51$.

Carlini CR, Grossi-de-Sa MF. Plant toxic proteins with insecticidal properties. A review on their potentialities as bio-insecticides. Toxicon $2002 ; 40: 1515-9$

Cherqui A, Tjallingii WF. Salivary proteins of aphids, a pilot study on identification, separation and immunolocalisation. J Insect Physiol tion and immunoloca

Couty A, Jouanin L, Pham Delegue $\mathrm{MH}$ Impact de protéines d'origine végétale exprimées dans des plantes transgéniques sur des insectes pollinisateurs et auxilliaires. In : Regnault-Roger $C$, Philogène $B$, Vincent $C$, eds. Biopesticides d'origine végétale. Paris Lavoisier, 2002.

Deraison C, Darboux I, Duportets L, et al. Cloning and characterization of a gut specific cathepsin L from the aphid Aphis gossypii. Insect Mol Biol $2004 ; 13$ : 165-7.

Devonshire AL, Field LM, Foster SP, et al. The evolution of insecticide resistance in the peach-potato aphid, Myzus persicae. Philos Trans R Soc Lond B Biol Sci 1998; 353 : 167784.

Down RE, Ford L, Woodhouse SD, et al. Tritrophic interactions between transgenic potato expressing snowdrop lectin (GNA), an aphid pest (peach-potato aphid; Myzus persicae (Sulz.)) an a beneficial predator (2-spot ladybird; Adalia bipunctata L.). Transgenic Res 2003: $12: 229-31$.

Du Y, Poppy GM, Powell W, et al. Identification of semiochemicals released during aphid feeding that attract parasitoid Aphidius ervi. $J$ Chem Ecol 1998 ; 24 : 1355-62.

Duvauchelle S, Dubois L. Évolution sanitaire des cultures de pommes de terre en 1997 : I'épidémie de mildiou la plus virulente depuis 1981: betteraves et pommes de terre (1997 potato crops-health review: the harshest outbreaks of late blight since 1981). Phytoma Def Veg $1997 ; 502$ : 24-6.

Eichenseer $\mathrm{H}$, Mathews MC, Bi JL, et al. Salivary glucose oxidase: multifunctional roles for Helicoverpa zea?. Arch Insect Biochem Physiol $1999 ; 42$ : 99-109.

Eigenbrode SD, Ding $\mathrm{H}$, Shiel $\mathrm{P}$, et al. Volatiles from potato plants infected with potato leaf Myzus persicae (Homoptera: Aphididae). Proc $R$ Soc Lond B Biol Sci 2002 ; 269 : 455-60.

Esposito F, Fogliano V, Cardi T, et al. Glycoalkaloid content and chemical composition of potatoes improved with non-conventional breeding approaches. J Agr Food Chem 2002 ; bo: 1553-61.

Ewen SWB, Pusztai A. Effect of diets containing genetically modified potatoes expressing Galanthus nivalis lectin on rat small intestine. Lancet 1999 ; 354 : 1353-4.
Fitches $\mathrm{E}$, Wilkinson $\mathrm{H}$, Bell $\mathrm{H}$, et al. Cloning, expression and functional characterisation of chitinase from larvae of tomato moth (Lacanobia oleracea): a demonstration of the insecticidal activity of insect chitinase. Insect Biochem Molec 2004 ; 34 : 1037-50.

Flanders KL, Hawkes JG, Radcliffe EB, et al. Insect resistance in potatoes: sources, evolutionary relationships, morphological and chemical defenses, and ecogeographical associations. Euphytica $1992 ; 61$ : 83-91.

Foster SP, Young S, Williamson MS, et al. Analogous pleiotropic effects of insecticide resistance genotypes in peach-potato aphids and houseflies. Heredity $2003 ; 91$ : 98-106.

Fragoyiannis DA, McKinlay MG, Mello JPFD. Studies of the growth, development and reproductive performance of the aphid shape Myzus persicae on artificial diets containing potato glycoalkaloids. Entomol Exp Appl potato glycoalkato 198 : 59-66.

Francis F, Lognay G, Haubruge E. Olfactory responses to aphid and host plant volatile releases: (E)- $\beta$-farnesene an effective kairomone for the predator Adalia bipunctata. J Chem Ecol $2004 ; 30$ : 741-5.

Francis F, Martin T, Lognay G, et al. Role of (E)$\beta$-farnesene in systematic aphid prey location
by Episyrphus balteatus larvae. Eur J Entomol $2005 ; 102: 431-6$.

Francis F, Harmel N, De Pauw E, Haubruge E. Proteomic approach to investigate aphid-plant interactions. J Insect Sci $2006 ; 6$ : 20-1.

Friedman M, McDonald GM. Potato glycoalkaloids: chemistry, analysis, safety, and plant physiology. Crit Rev Plant Sci 1997 ; 16 : 55-62.

Gatehouse AMR, Davison GM, Stewart JN, et al. Concanavalin A inhibits development of tomato moth (Lacanobia oleracea) and peachpotato aphid (Myzus persicae) expressed in transgenic potato plants. Mol expressed in transgen
Breed $1999 ; 5: 153-5$.

Gibson RW, Pickett JA. Wild potato repels aphids by release of aphid alarm pheromone. Nature $1983 ; 302$ : 608-9.

Goggin FL, Williamson VM, Ullman DE. Variability in the response of Macrosiphum euphorbiae and Myzus persicae (Hemiptera: Aphididae) to the tomato resistance gene Mi. Environ Entomol $2001 ; 30: 101-6$.

Güntner C, Vazquez A, Gonzalez G, et al. Effect of Solanum glycoalkaloids on potato aphid Macrosiphum euphorbiae: part II. J Chem Ecol $2000 ; 26: 1113-21$.

Haq SK, Atif SM, Khan RH. Protein proteinase inhibitor genes in combat against insects pests, and pathogens: natural and engineered phytoprotection. Arch Biochem Biophys 2004 431 : 145-9.

Harborne JB. Introduction to Ecological Biochemistry. Londres : Academic Press, 1993.

Harmel N, Almohamad R, Fauconnier ML, et al. Role of terpenes from aphid infested potato as indirect defence on Episyrphus balteatus behaviour. Insect Sci 2007 ; 14 : 57-63.

Harmel N, Létocart E, Cherqui A, et al. Diversity of aphid salivary proteins: a proteomic investigation on Myzus persicae. Insect $\mathrm{Mol}$ Biol $2008 ; 17(2): 165-74$.

Hildebrand D, Brown GC, Jackson DM, et al. Effects of some leaf-emitted volatile compounds on aphid population increase. J Chem Ecol 1993; 19:1875-7. 
Huang HC, Harper AM, Kokko EG, et al. Aphid transmission of Verticillium albo-atrum to alfalfa. Can J Plant Pathol $1983 ; 5$ : 141-7.

Kaloshian I, Walling LL. Hemipterans as plant pathogens. Annu Rev Phytopathol 2005 ; 43: pat-521.

Kehr J. Phloem sap proteins: their identities and potential roles in the interaction between plants and phloem-feeding insects. J Exp Bot 2006 ; $57: 767-74$.

Kennedy JS, Day MF, Eastop VF. A conspectus of aphids as vectors of plant viruses. New Phytol $1963 ; 62$ : 113-4.

Kikuchi K, Hirai M, Shiotsuki T. Molecular cloning and tissue distribution of farnesyl pyro-
phosphate synthase from the silkworm Bomphosphate synthase from the silkworm Bom167-82.

Lachman J, Hamouz K, Orsák M, Pivec V. Potato glycoalkaloids and their significance in plant protection and human nutrition. Series Rostlinná Výroba $2001 ; 47$ : 181-91.

Martinez de Ilarduya O, Xie QG, Kaloshian I. Aphid-induced defense responses in $\mathrm{Mi}$ - 1mediated compatible and incompatible tomato interactions. Mol Plant Microbe In $2003 ; 16: 699-708$

Mattiaci L, Dicke M, Posthumus MA. $\beta$-glucosidase: an elicitor of herbivore-induced plant odor that attracts host searching parasitic wasps. Proc Natl Acad Sci USA 1995; 92: 2036-40.

Miles PW. The responses of plants to the feeding of Aphidoidea: principles. In : Minks AK, Harrewijn P, eds. Aphids, their biology, natural enemies and control. Amsterdam (The Netherlands) : Elsevier, 1989.

Neal JJ, Tingey WM, Steffens JC. Sucrose esters of carboxylic acids in glandular trichomes of Solanum berthaultii deter settling and probing by the green peach aphid. J Chem Ecol $1990 ; 16: 487-97$
Ninkovic V, Al Abassi S, Pettersson J. The influence of aphid-induced plant volatiles on ladybird beetle searching behaviour. Biol Control $2001 ; 21: 191-5$

Paré PW, Tumlinson JH. Plant volatiles as a defense against insect herbivores. Plant Physiol $1999 ; 121$ : 325-32.

Pickett JA, Wadhams LJ, Woodcock CM, et al. The chemical ecology of aphids. Annu Rev Entomol $1992 ; 37: 67-90$

Powell G, Tosh CR, Hardie J. Host plant selection by aphids: behavioral, evolutionary and applied perspectives. Annu Rev Entomol $2006 ; 51: 309-30$.

Rahbé Y, Deraison C, Bonadé-Bottino M, et al. Effects of the cysteine protease inhibitor oryzacystatin (OC-1) on different aphids and reduConce of Myzus persicae on OC-1 expressing transgenic oilseed rape. Plant Sci $2003 ; 164: 441-50$

Rahbé Y, Ferrasson E, Rabesona H, Quillien L. Toxicity to the pea aphid Acyrthosiphon pisum of anti-chymotrypsin isoforms and fragments of Bowman-Birk protease inhibitors from pea seeds. Insect Biochem Molec 2003 ; 33 : 299 306.

Rangarajan A, Miller AR, Veilleux RE. Leptine glycoalkaloids reduce feeding by Colorado potato beetle in diploid Solanum sp. hybrids. potato beetle in diploid Solanum sp. hy

Saguez J, Hainez R, Cherqui A, et al. Unexpected effects of chitinases on the peach-potato aphid (Myzus persicae Sulzer) when delivered via transgenic potato plants (Solanum tuberosum Linné) and in vitro. Transgenic Res 2005 ; 14 : 57-67.

Saguez J, Dubois F, Vincent C, et al. Differential aphicidal effects of chitinase inhibitors on the polyphagous homopteran Myzus persicae the polyphagous homopteran Myzus persica
(Sulzer). Pest Manag Sci 2006 ; $62: 1150-4$.

Sauvion N, Charles H, Febvay G, Rahbé Y. Effects of jackbean lectin (ConA) on the feeding behaviour and kinetics of intoxication of the pea aphid, Acyrthosiphon pisum. Entomol Exp App/ $2004 ; 110: 31-4$
Schoonhoven LM, Jermy T, Van Loon JJA. Insect-Plant Biology. London: Chapman \& Hall, 1998.

Storer JR, Powell G, Hardie J. Settling responses of aphids in air permeated with non-host plant volatiles. Entomol Exp Appl 1996; 80 : plant

Tingey WM, Laubengayer JL. Defense against the green peach aphid and potato leafhopper by glandular trichomes of Solanum berthaultii. J Econ Entomol 1981 ; 74 : 721-5.

Tjallingii WF, Hogen Esch TH. Fine structure of aphid stylet routes in plant tissues in correlation with EPG signals. Physiol Entomol 1993 ; $18: 317-8$.

Vallejo RL, Collins WW, Schiavone RD. Genetics and incorporation of glandular trichomes and polyphenol oxidase activity into an advanced Solanum phureja-S. stenotomum diploid potato population. J Am Soc Hortic Sci 1994 ; $119: 824-8$.

Vaananen T, Kuronen P, Pehu E. Comparison of commercial solid-phase extraction sorbents for the sample preparation of potato glycoalkaloids. J Chromatogr A 2000 ; 869 : 301-5.

Van Damme EJM, Peumans WJ, Pusztai A Bardocz S. Handbook of plant lectins: properties and biochemical applications. Chichester (Grande-Bretagne) : John Wiley and Sons Ltd, 1997.

Vandermoten S, Charloteaux B Santini S et al. Characterization of a novel aphid prenyl transferase displaying dual geranyl/farnesyl transferase displaying dual geranyl/farnesyl
diphosphate synthase activity. FEBS Lett 2008 ; 582 : 1928-34.

Wolfson JL, Murdock LL. Diversity in digestive proteinase activity among insects. J Chem Ecol $1990 ; 16: 1089-102$.

Ying SH, Feng MG, Xu ST. Field efficacy of emulsifiable suspensions of Beauveria bas siana conidia for control of Myzus persicae population on cabbage. Chin J Appl Ecol $2003 ; 14: 545-8$ 